\title{
Role of self-focussed reappraisal of negative emotion in emergence of emotional symptoms in adolescent girls
}

\author{
Gin S. Malhi, Pritha Das, Tim Outhred, Richard A. Bryant and Vince Calhoun
}

\section{Background}

Adolescent subthreshold emotional symptoms arise from impaired self-referential information-processing and approachavoidance behaviour network integration, which compromises goal evaluation and pursuit strategies.

\section{Aims}

We investigated whether impairment of negative emotion (goal) reappraisal strategies (self-focussing and self-distancing) generates emotional symptoms (emotional disorders precursors).

\section{Method}

Using functional magnetic resonance imaging and a triple-network model (default mode, executive control and salience), functional connectivity differences within and between networks, and their modulation by task and relationships with emotional symptoms were determined in healthy adolescent girls $(N=202)$ grouped by presence or absence of emotional symptoms.

\section{Results}

The groups differed in spectral power distribution and in dorsal default mode network and right executive control network modulation when self-focussing and self-distancing, respectively. Girls without emotional symptoms had greater spectral power and less network modulation. Greater spectral power was associated with reduced emotional symptoms and less dorsal default mode network modulation when self-focussing.

\section{Conclusions}

The early phases of anxiety and depressive disorders in adolescence are marked by emotional symptoms that usually emerge in the context of negative life events. To contend with the negative effect of such events, a typical reappraisal strategy is to distance oneself and switch the focus of one's thinking. This brain-imaging study in adolescent girls prone to the development of emotional disorders has found functional changes in key neural networks that are involved in reappraisal and shown that this process is impaired. This is important because it provides an early indication of these common disorders and a potential target for psychological interventions.

\section{Declaration of interest}

G.S.M. has received support from AstraZeneca, Eli Lily \& Co., Organon, Pfizer, Servier and wyeth; has been a speaker for AstraZeneca, Eli Lily \& Co., Janssen Cilag, Lundbeck, Pfizer, Ranbaxy, Servier and wyeth; and has been a consultant for AstraZeneca, Eli Lily \& Co., Janssen Cilag, Lundbeck and Servier. P.D., T.O., R.A.B. and V.C. have no conflicts of interest to report.

\section{Keywords}

Adolescent; adolescence; emotion regulation; default mode network; executive control network.

\section{Copyright and usage}

(c) The Authors 2020.
A study comprising 12395 adolescents from 11 countries found the prevalence of subthreshold anxiety and depression (emotional disorders) in adolescents to be as high as 32 and $29.2 \%$, respectively. ${ }^{1}$ These subthreshold emotional symptoms have prognostic significance and may be able to serve as predictors of disorders later in life. ${ }^{2,3}$ Importantly, emotional symptoms are not only associated with an increased burden of disease, but also with an increased risk of suicide. ${ }^{1}$ Recent findings from our own research in adolescent girls, suggest that these emotional symptoms emerge as a result of impaired integration between networks involved in self-referential information-processing and approach-avoidance behaviours. ${ }^{4}$ We have also suggested that this impairment perhaps also compromises the evaluation of goals and strategies to pursue them, and thereby leads to a failure to achieve desired outcomes, and that this is an antecedent to depression. ${ }^{4}$

An individual's emotional response, particularly to an aversive event, can be altered by emotion regulation strategies such as reappraisal, ${ }^{5}$ which has been found to be one of the most flexible and efficient means of diminishing the negative effect of an aversive event. ${ }^{6,7}$ However, improper use of reappraisal can make an already negative situation much worse. ${ }^{7}$ For example, when reappraising a negative situation if an individual adopts the wrong strategy (i.e. appraises negative content and relates it to their own person and experience, known as reappraisal using a self-focussing/self-referential approach), they may in fact reinforce negative views of themselves and this may exacerbate their emotional symptoms and sink them further into depression. This is why depressed patients are thought to benefit clinically by reframing (reappraising) negative emotional situations from a perspective that is more distant from themselves (self-distancing). ${ }^{8}$

\section{Reappraisal in adolescents}

Although increasing attention has been paid to reappraisal in adults, there are relatively few studies, to our knowledge, that have investigated reappraisal by using neuroimaging in adolescents. ${ }^{9-12}$ These handful of studies have investigated the reappraisal of negative emotions, but critically they differ with respect to the question they have addressed and the age group they have defined as adolescence. Furthermore, these studies have primarily examined the self-distancing aspect of reappraisal of negative emotions. No study, thus far, has investigated neural underpinning of both self-focussing (which augments negative emotion) and self-distancing (which diminishes negative emotion) strategies to reappraise negative emotion to understand emergence of emotional symptoms in adolescents.

Another key difference is that all of the earlier neuroimaging studies investigating reappraisal in adolescents have used the general linear model (GLM) for their analyses. However, the field as a whole is increasingly attaching greater importance to interrogating brain function from a network perspective in which distributed brain regions work in concert. These temporally coherent networks are usually identified from functional magnetic resonance 
imaging (fMRI) data by independent component analysis, ${ }^{13,14}$ and employing this method has several advantages over GLM. For instance, it reduces the number of comparisons needed, is more sensitive to between-group differences and is able to identify differences not discernible by GLM. ${ }^{14,15}$ Moreover, it has been suggested that functional networks that subserve complex cognitive capabilities undergo protracted developmental changes over the first two decades of human life, ${ }^{16}$ and that the analysis of temporally coherent networks is capable of differentiating between healthy and abnormal brain function. ${ }^{14}$ Hence, a network approach is ideally suited to investigating the neural mechanisms underlying the reappraisal of negative emotions in adolescents. This is particularly important because a mechanistic understanding of the processes that underpin emotional symptoms, which in turn are likely to lead to emotional disorders, is crucial for the identification of therapeutic targets and for the development of effective treatments involving both medications and psychological interventions.

\section{Aims}

Therefore, the present study investigated the reappraisal of negative images in adolescent girls (aged 12-18 years) comparing selffocussed (shifting viewpoint to consider the situation from a firstperson perspective, thus augmenting negative emotion) and selfdistancing (perceiving the situation from a third-person perspective, thus diminishing negative emotion) approaches. ${ }^{7}$ A well-known triple-network model that consists of the default mode network $(\mathrm{DMN})$, executive control network (ECN) and salience network was adopted for imaging data analysis. ${ }^{17}$ Adoption of this model is important because aberrant switching between DMN-mediated self-referential and ECN-mediated goal-directed cognitive processes is thought to contribute to major depressive disorder, ${ }^{18}$ and our earlier resting-state fMRI findings in mid-adolescent (aged 14-15 years) girls show that intrinsic functional connectivity between these networks (FNC) differentiates those girls who are vulnerable to emotional disorders from those who are not. ${ }^{4}$

Hence the present study examined FNC both within (spatial maps and spectral power) and between these key networks (functional network connectivity), using a multivariate-testing framework $^{19}$ to understand emotional symptoms as result of impairment in reappraising negative emotions in adolescents known to be vulnerable to emotional disorders.

\section{Method}

\section{Participants}

In total, 228 adolescent girls (aged 12-18 years) from a girls school in Sydney participated in this study. On the same day participants underwent neuroimaging and completed a series of self-report questionnaires at the CADE Clinic (www.cadeclinic.com). Participants with a history of significant neurological illness (e.g. traumatic head injury, loss of consciousness, epilepsy or other neurological event), developmental disability or any medical/physical condition that precluded them from neuroimaging were excluded. Written informed consent was obtained from the parents/guardians and the principal of the school, and the study was approved by the hospital Human Research Ethics Committee at Royal North Shore Hospital (approval number HREC/11/HAWKE/59).

Following our earlier research, ${ }^{4,20}$ participants were divided into two groups (with and without emotional symptoms), using Child and Adolescent PsychProfiler (CAPP). ${ }^{21}$ Participants qualified as having emotional symptoms if they surpassed the cut-off score for any mental disorder according to CAPP, or had a history of a mental health issue for which they had received psychological support or treatment.

\section{Experimental procedure}

The experimental procedure comprised four phases. The first phase involved assessment of the level of emotional symptoms for which participants completed a series of self-reported questionnaires. Depression was measured using the Children's Depression Inventory (CDI), ${ }^{22}$ anxiety was measured using the State-Trait Anxiety Inventory (STAI) ${ }^{23}$ and emotion regulation was measured using the Difficulties in Emotional Regulation Scale (DERS). ${ }^{24}$ The second phase involved training and testing in emotion regulation techniques. The participants rehearsed outside the scanner to familiarise themselves with the reappraisal strategies. Negative emotional stimuli selected from the International Affective Pictures System (IAPS) ${ }^{25}$ were presented (see Supplementary IAPS file available at https://doi.org/10.1192/bjp.2019.255) and participants were instructed to either attend to the visual stimuli (i.e. not to apply any reappraisal strategy) or to reappraise (switching between selffocussed and self-distanced) their emotional reactions to each picture. In the increase condition, the self-focussed perspective was used and participants were instructed to think that the scene was 'real', that it does affect them and furthermore the situation was going to worsen. In the decrease condition, a self-distancing perspective was used and participants were instructed to think that the scene was not real, that it does not affect them and that the situation was going to improve. In the watch condition, participants were asked to simply view the image be it negative or neutral. If necessary, the experimenter gave further verbal instructions to shape the emotion regulation strategy and the training session was repeated. The third phase involved performing the emotion regulation task within the scanner. The IAPS images used for practise were different from those used during scanning. Finally, the fourth phase involved a formal debriefing.

\section{fMRI task}

The negative emotion regulation task comprised 45 negative and 15 neutral IAPS images. The task had four conditions: participant had to increase/upregulate (NegInc) (using self-focussing approach), or decrease/downregulate ( $\mathrm{NegDec}$ ) (using self-distancing approach) their negative emotions in response to negative images, or they had to just watch (no regulation) negative (WatNeg) or neutral (WatNeut) images. Images were presented for $6 \mathrm{~s}$ and each condition was preceded by a cue (increase or decrease or watch) for $2 \mathrm{~s}$, followed by the presentation of a rating scale slide for $3 \mathrm{~s}$. During presentation of the rating slide participants had to press a button to indicate the magnitude of the negative emotion they felt, ranging on a scale from 1 , weak to 5 , strong. Conditions were presented in an event-related fashion and in-between images a fixation cross was presented with jittered duration $(2.5,5$ or $7.5 \mathrm{~s})$.

\section{Imaging data acquisition}

Following our earlier protocol, ${ }^{4}$ functional imaging data was acquired on a University of Sydney 3T Siemens Magnetom Trio Scanner based at the Advanced Research and Clinical High-field Imaging facility. A $\mathrm{T}^{*}$-weighted, gradient-echo echo-planner imaging sequence (29 axial slices, slice thickness $4 \mathrm{~mm}$ with $1 \mathrm{~mm}$ gap, repetition time $2000 \mathrm{~ms}$, echo time $35 \mathrm{~ms}$, flip $70^{\circ}, 64 \times 64$ matrix) was used to acquire 485 whole-brain volumes of functional data. Head motion during scanning was restrained by foam pads inserted on each side. 


\section{Data pre-processing}

Each participant's functional images were first inspected visually for scanner artefacts and gross anatomical abnormalities, and then reoriented so that the origin of the image lay within $3 \mathrm{~cm}$ of the anterior commissure. The initial five images were discarded to remove longitudinal equilibration effects. The imaging data was then pre-processed with the statistical parametric mapping software SPM8 (MATLAB for Windows, Wellcome Trust; http://www.fil.ion. ucl.ac.uk/spm/software/spm8). Images were first realigned with INRIAlign, version 1.01 (MATLAB for Windows, Inria; http:// www-sop.inria.fr/epidaure/software/INRIAlign/), a motion correction algorithm unbiased by local signal changes, and then corrected for slice-time variation, using the middle slice as the reference frame. Then, these images were spatially normalised to a common stereotactic space with the Montreal Neurological Institute echoplanner imaging template, and spatially smoothed with a Gaussian kernel of $8 \mathrm{~mm}^{3}$ full width at half maximum. Following spatial normalisation, the data (originally acquired at $3.75 \times$ $3.75 \times 5 \mathrm{~mm}^{3}$ ) were slightly subsampled to $3 \times 3 \times 3 \mathrm{~mm}^{3}$, resulting in $53 \times 63 \times 46$ voxels. Exclusion criteria regarding head motion were applied and participants were excluded from analysis if their functional scans showed extreme head motion (translation $>2 \mathrm{~mm}$ and rotation $>1^{\circ}$ ).

\section{Data post-processing}

From the pre-processed data of all participants, 14 networks covering the majority of cortical and subcortical grey matter ${ }^{26}$ were identified with the masks from the Functional Imaging in Neuropsychiatric Disorders Laboratory (https://findlab.stanford. edu/functional_ROIs.html) and spatially constrained group independent component analysis, as implemented within the GIFT software (version 4.0b; MATLAB for Windows, Mind Research Network; http://mialab.mrn.org/software/gift/). Then from these 14 networks, three (DMN, ECN and salience network) were chosen as our networks of interest. To represent DMN, dorsal default mode network ( $\mathrm{dDMN}$ ) and ventral default mode network (vDMN) masks were chosen. Similarly, ECN was represented by both left executive control network (lECN) and right executive control network (rECN) masks, and $\mathrm{SN}$ by anterior salience network (aSN) and posterior salience network (pSN) masks. Participant-specific spatial maps and time courses were obtained by spatiotemporal regression. For each participant, time courses from these networks were detrended, orthogonalised with respect to their head motion parameters (estimated during realignment stage of pre-processing) and de-spiked with a third-order spline.

\section{Outcome measures}

To investigate differences between groups (with and without emotional symptoms), four outcome variables were investigated: the spatial map of a network where voxel intensity in the spatial map dictates the correspondence between a voxel time course and a network time course, and thereby provides a measure of co-activations/synchronisation (strength of connectivity) in a region within a given network; the power spectra of a network, which reveals frequency-based coherence measures within a network; FNC and modulation of networks by the task.

Before comparing any network's spatial map, each spatial map was thresholded so that it includes only those subsets of voxels that have shown strong and consistent activation across participants and therefore are most likely to represent the network. The threshold was based on the distribution of voxel-wise $t$-statistics and voxels exceeding the threshold on the right tail $(t>$ mean +4 s.d.) were selected. These thresholded spatial maps were subsequently compared between groups.

To determine differences between groups in a network's spectral power, its time course was transformed to 257 spectral bins using fast Fourier transform and then the power in each bin was compared.

Before comparing FNC, the time course data associated with the components were filtered with a band-pass Butterworth filter with cut-off frequencies at $0.01 \mathrm{~Hz}$ and $0.15 \mathrm{~Hz}$. Then, the procedure described by Jafri et $a l^{27}$ was used to determine FNC. For each participant, correlations between pairwise combinations were calculated with Pearson's correlations, and resultant $r$-values were transformed to $z$-scores with Fisher's transformation as a standardised measure of FNC.

The MANCOVAN utility within GIFT was used to determine differences between groups in these measures (spatial map, power spectra and FNC) and results were corrected for multiple comparisons with the false discovery rate at $P<0.05$.

To understand how the task modulates functioning of these chosen networks, a regression analysis was performed on the network's time courses with the GLM design matrix created with SPM8. The design matrix consisted of four regressors representing four experimental conditions (NegInc, NegDec, WatNeg and WatNeut) and their temporal derivatives. This resulted in a set of beta weights, representing the degree to which a network was modulated by a particular task relative to the fixation baseline in each participant. Downregulation of negative emotion was calculated by subtracting beta weights associated with WatNeg and NegDec (NegDec-WatNeg). Similarly, upregulation of negative emotion was calculated by subtracting beta weights associated with WatNeg and NegInc (NegInc-WatNeg). Another contrast from beta weights associated with WatNeg and WatNeut (WatNegWatNeut) was created to investigate the effect of no regulation of negative emotion.

To investigate whether there were differences between groups in modulation of chosen networks during regulation (up or down) or no regulation of negative emotion, the resultant beta weights for each chosen network were entered into a general linear multivariate analyses model in SPSS version 25 for Windows (IBM) as dependent variables and group (with and without emotional symptoms) as a fixed factor. Identified significant $(P<0.05)$ differences were followed by two-sample $t$-tests for directionality.

\section{Post-hoc analyses}

Relationship of the spectral power in high-frequency range $(0.2-0.24 \mathrm{~Hz})$ with task modulation and emotional symptoms

To understand whether observed differences in the modulation of networks by upregulation (dDMN) and downregulation (rECN) of negative emotion are because of differences in spectral power in the high-frequency range in the four identified networks ( $\mathrm{rECN}, \mathrm{lECN}, \mathrm{dDMN}$ and $\mathrm{vDMN}$ ), the spectral power of these networks in high-frequency range $(0.2-0.24 \mathrm{~Hz})$ was correlated with the beta weights associated with the $\mathrm{dDMN}$ during upregulation, and the rECN during downregulation, of negative emotion.

Spectral powers were also correlated with subthresholded emotional symptoms (DERS, state anxiety, trait anxiety, and CDI) to understand relation between spectral power and emotional symptoms.

\section{Results}

\section{Participants}

Out of 228 participants 26 participants' data were excluded because of head movement, and therefore the final analysis is based on 202 
Table 1 Emotional symptoms groups differed significantly in age and subsyndromal emotional symptoms such that age and scores for dysregulation of emotion (DERS), state and trait anxiety and depression (CDI) were higher in in those with emotional symptoms compared with those without

\begin{tabular}{|c|c|c|c|c|c|c|c|}
\hline & \multicolumn{7}{|c|}{$t$-Test for equality of means } \\
\hline & \multirow[b]{2}{*}{$t$} & \multirow[b]{2}{*}{ d.f. } & \multirow[b]{2}{*}{ Significance (two-tailed) } & \multirow[b]{2}{*}{ Mean difference } & \multirow[b]{2}{*}{ s.e. Difference } & \multicolumn{2}{|c|}{$95 \% \mathrm{Cl}$ of the difference } \\
\hline & & & & & & Lower & Upper \\
\hline Age (years) & -2.156 & 196.16 & 0.032 & -0.518 & 0.024 & -0.099 & -0.044 \\
\hline DERS Total score & -7.201 & 166.13 & $<0.0001$ & -21.145 & 2.936 & -26.942 & -15.347 \\
\hline State anxiety score & -6.553 & 165.52 & $<0.0001$ & -8.237 & 1.257 & -10.718 & -5.755 \\
\hline Trait anxiety score & -7.946 & 152.78 & $<0.0001$ & -11.167 & 1.405 & -13.943 & -8.39 \\
\hline CDI total score & -8.474 & 135.25 & $<0.0001$ & -8.063 & 0.951 & -9.944 & -6.181 \\
\hline
\end{tabular}

participants (88 in the group with emotional symptoms, and 114 in the group without emotional symptoms).

There was no significant difference in age between groups, but subthreshold emotional symptoms differed (Table 1). As can be seen from the table, the group with emotional symptoms had significantly greater levels of psychopathology with greater mood and anxiety symptoms, and lower emotion regulation, compared with those without emotional symptoms.

\section{Behavioural data}

To investigate whether ratings for the increase, decrease and watch conditions were different between groups, a GLM multivariate analyses was performed in SPSS where ratings for the four conditions (increase, decrease, watch negative and watch neutral) were entered as dependent variables and group was a fixed factor. Groups did not differ in ratings in any one of these four conditions. In general, the overall rating was higher for the NegInc condition $(4.29 \pm 0.45)$ followed by the WatNeg $(3.56 \pm 0.69)$, NegDec $(2.55$ $\pm 0.74)$ and WatNeut $(1.57 \pm 0.49)$ conditions.

\section{Imaging data}

Spatial maps of our networks of interest are shown in Fig. 1 and primary regions within each network are provided in Supplementary Table 1. There were no significant differences between groups in spatial maps and FNCs. The distribution of spectral power differed between groups such that those without emotional symptoms had more power, compared to those with emotional symptoms, in the high-frequency range $(0.2-0.24 \mathrm{~Hz})$ in the $\mathrm{IECN}, \mathrm{rECN}, \mathrm{dDMN}$ and $\operatorname{vDMN}$ (Fig, 2).

Groups also differed significantly in terms of the extent to which there was modulation of the networks by the task condition. Groups differed significantly during upregulation of emotion (using self-focussing approach) in modulation of the $\mathrm{dDMN}(t=-2.13$, $P=0.034)$ and the $\operatorname{rECN}(t=-1.97, P=0.049)$ during downregulation of emotion (using the self-distancing approach). In both cases, modulation was greater in the group with emotional symptoms compared with those without. There were no differences between the groups when there was no regulation of emotion.

\section{Post-hoc analyses}

Relationship between high-frequency $(0.2-0.24 \mathrm{~Hz})$ spectral power, task modulation and subclinical symptoms

Relationships have been summarised in Table 2. The spectral power of the lECN $(r=-0.204, P=0.004), \mathrm{rECN}(r=-0.161, P=0.022)$ and $\operatorname{dDMN}(r=-0.139, P=0.049)$ showed significant negative correlation with the effect size of $\mathrm{dDMN}$ modulation produced by upregulation of emotion during self-focussed condition. Spectral power of these same networks showed significant negative correlation with scores of depression (measured by CDI), state and trait anxiety (measured by STAI) and emotion dysregulation (measured by DERS). In contrast, the spectral power of the vDMN showed negative correlation only with CDI and trait anxiety. These relationships suggest that decrease in spectral power increases emotional symptoms and also enhances self-referential processing.

\section{Discussion}

The current study is the first, to our knowledge, to investigate the neural underpinnings of negative emotion reappraisal, using both augmentation and diminution of negative emotions to understand de-emergence of emotional symptoms in adolescents known to be vulnerable to emotional disorders. The findings support the results of our earlier study in which we implicated the ECN and DMN in the emergence of emotional symptoms in these adolescents, and also extends our knowledge by suggesting that impaired integration among these networks likely contributes to self-focussed reappraisal of negative events.

Previous neurocognitive research suggests that the reappraisal of negative emotion through self-focussed/self-referential processing engages midline circuitry, ${ }^{7}$ whereas detaching oneself engages the superior frontal gurus. ${ }^{28}$ In line with this hypothesis, the selffocussed condition in our study modulated activity of the dDMN, which encompasses midline brain regions, whereas the self-distancing condition modulated activity of the rECN, which encompasses the superior frontal gyrus. Groups differed in these modulations, and in both conditions these modulations were greater in those with emotional symptoms compared with those without. Interestingly, a significant positive correlation $(r=0.204, P=0.004)$ was found between the modulation of these networks such that those girls in whom there was greater modulation of the $\mathrm{dDMN}$ when engaged in self-focussed processing, also required greater modulation of the rECN when engaged in the self-distancing condition. This suggests that self-focussed thought-processing is always present to some extent, and is perhaps the default state. It also suggests that in those with emotional symptoms, this default state is more pronounced and hence greater effort is necessary to disengage and switch from this state of self-focussed processing to instead employ and re-engage with a self-distancing approach, as per the demand of the task. This is clinically relevant because aberrant switching between DMN-mediated self-referential and ECNmediated goal-directed cognitive processes is purported to be at the heart of difficulties experienced by patients with major depressive disorder when attempting to shift attention from self-focussed thought-processing and -orienting to goal-directed behaviour. ${ }^{18}$ It is therefore a core process that both contributes to, and can counter the effects of depression.

Once again, in the present study we found that the same networks (DMN and ECN) that differentiated mid-adolescent girls (aged 14-15 years) with respect to emotional symptoms in our 
dDMN

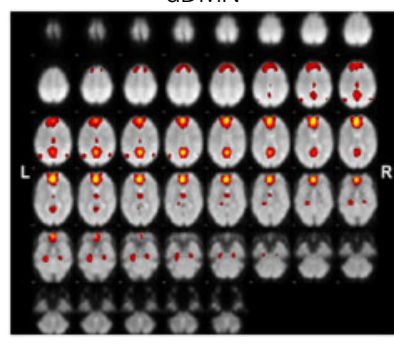

rECN

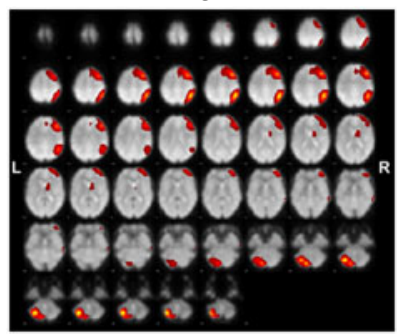

VDMN

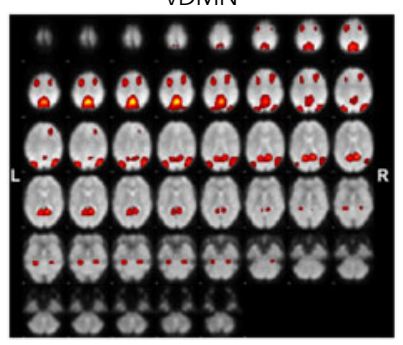

aSN

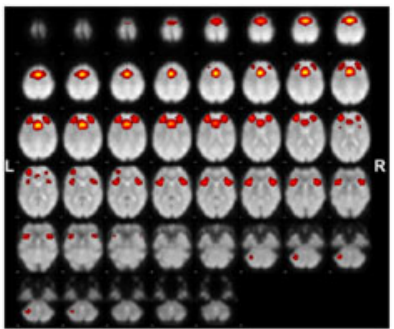

IECN

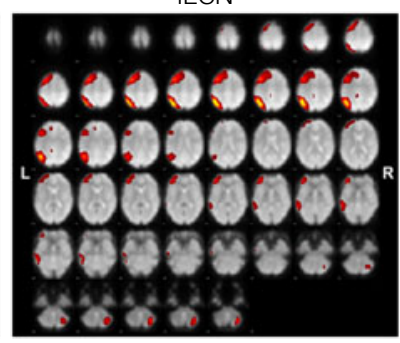

pSN

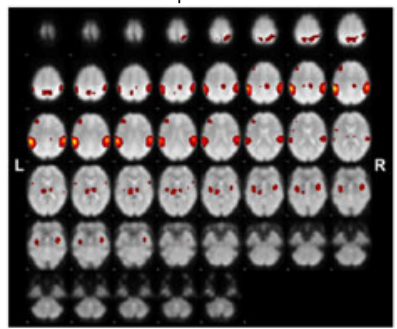

Fig. 1 Spatial maps of chosen networks.

aSN, anterior salience network; dDMN, dorsal default mode network; IECN, left executive control network; pSN, posterior salience network; rECN, right executive control network; VDMN, ventral default mode network.

earlier study ${ }^{4}$ showed significant differences in spectral power between those with and without emotional symptoms. To date, most studies using fMRI have investigated the oscillatory activity of the blood oxygenation level-dependent (BOLD) signal in the low-frequency range $(<0.1 \mathrm{~Hz})$ to understand activity patterns of the resting brain. And thus far, only a few studies have looked into this oscillatory activity as a function of power, within various frequency bands of the fMRI signal using a task. ${ }^{29,30}$ For example, Balsters et al found that task accuracy was inversely proportional to the BOLD spectral power in the higher-frequency band, such that greater power was associated with poorer performance reflected

Significant effects of group with and without emotional symptoms $(P<0.05)$

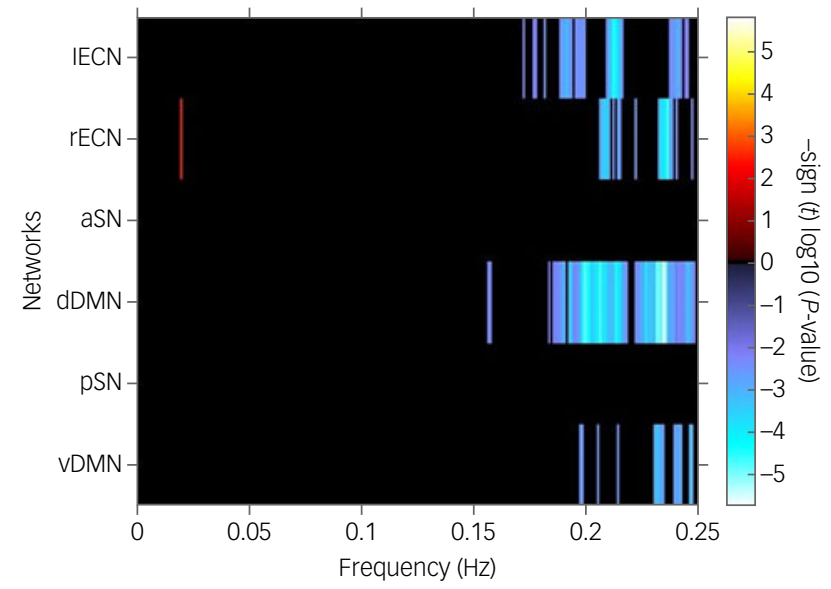

Fig. 2 Differences between groups (with/without emotional symptoms) in the distribution of spectral power. Those without emotional symptoms had more power, compared with those with emotional symptoms, in the high-frequency range $(0.2-0.24 \mathrm{~Hz})$ in the left (IECN) and right (rECN) executive control networks, in the anterior (aSN) and posterior (pSN) salience networks, and also in the dorsal (dDMN) and ventral (vDMN) default mode networks. by slower reaction time. ${ }^{29}$ But findings from Baria et al suggest that brain regions involved in more complex information-processing should exhibit more power in higher-frequency BOLD oscillations. ${ }^{30}$ In our study, we did not find any correlation between spectral power and behavioural data (rating of images). However, high-frequency spectral powers of $1 \mathrm{ECN}, \mathrm{rECN}$ and $\mathrm{dDMN}$ showed negative correlations with task modulation of the dDMN during the self-focussing condition (but not with the rECN during self-distancing condition), and also behaviourally with the emotional symptoms the participants reported (CDI, DERS, state and trait anxiety), underscoring further the involvement of this network in the generation of emotional symptoms.

Our previous study suggested that impaired integration between self-referential (DMN) and approach-avoidance behavioural (ECN) systems, observed in mid-adolescent girls, may impair their judgement to adopt appropriate strategies to achieve their goals, and that it is this failure alongside other deficits that renders them vulnerable to depression. ${ }^{4}$ The findings from the present study not only corroborate the involvement of these networks in the development of emotional symptoms in adolescence, but also provide depth to our understanding. It suggests that emotional symptoms perhaps arise because of difficulties in being able to disengage from self-focussed reappraisal strategies and to enable engagement of self-distancing strategies when attempting to negate the impact of negative events. Impaired reappraisal capacity in depressed patients has been suggested to be because of dysfunctional approach-avoidance motivation. ${ }^{31}$ In line with this, our findings also show that networks underlying approach-avoidance behaviour contribute to impaired reappraisal of negative emotion in adolescents known to be vulnerable to emotional disorders.

In conclusion, the findings from this study suggest that emotional symptoms, which are an early sign of anxiety and depressive disorders, may arise in adolescents when faced with negative life events, in part because they adopt ineffective reappraisal strategies. Specifically, instead of using a self-distancing approach they select a self-focussing approach, and this then limits their ability to achieve their desired outcome, which in turn may be conducive to the development of depression. 
Table 2 Displaying predictors of subsyndromal emotional symptoms (DERS, CDI, state and trait anxiety) at different stages of adolescence (early, mid and late)

\begin{tabular}{|c|c|c|c|}
\hline Emotional symptoms & Early & Mid & Late \\
\hline $\mathrm{CDI}$ & & dDMN (beta $=-0.323, t=-2.71, P=0.009$ ) & rECN (beta $=-0.261, t=-2.29, P=0.025)$ \\
\hline DERS & $\begin{array}{l}\text { IECN (beta }=-0.273, t=-2.21 \\
\quad P=0.031)\end{array}$ & $\begin{array}{l}\mathrm{dDMN} \text { during upregulation (beta }=0.318 \\
\quad t=2.66, P=0.010 \text { ) }\end{array}$ & rECN (beta $=-0.267, t=-2.35, P=0.022)$ \\
\hline State anxiety & $\begin{array}{l}\text { IECN (beta }=-0.257, t=-2.08, \\
\quad P=0.042)\end{array}$ & $\begin{array}{l}\text { dDMN during upregulation (beta }=0.305 \\
\qquad t=2.54, P=0.014)\end{array}$ & \\
\hline Trait anxiety & $\begin{array}{l}\text { IECN (beta }=-0.290, t=-2.37 \\
\quad P=0.021)\end{array}$ & $\begin{array}{l}\mathrm{dDMN}(\mathrm{beta}=-0.323, t=-2.71 \\
\quad P=0.009)\end{array}$ & rECN (beta $=-0.251, t=-2.219, P=0.031)$ \\
\hline \multicolumn{4}{|c|}{$\begin{array}{l}\text { These relationships suggest impairment in central executive networks (IECN and rECN) and self-referential processing network (dDMN) underlie emotional symptoms in adolescents but } \\
\text { particular relationship vary according to the stages of adolescence. In early and late adolescence it is impairment in the regulatory networks (IECN and rECN), whereas in the mid-ado- } \\
\text { lescence it is in the self-referential processing network (dDMN). } \\
\text { DERS, Difficulties in Emotional Regulation Scale; CDI, Children's Depression Inventory; State anxiety, the state anxiety scale from the State-Trait Anxiety Inventory; Trait anxiety, the trait } \\
\text { anxiety scale from the State-Trait Anxiety Inventory; dDMN, dorsal default mode network; rECN, right executive control network, IECN, light executive control network. }\end{array}$} \\
\hline
\end{tabular}

\section{Limitations}

Although the findings from this study provide some novel insights into the emergence of emotional symptoms in adolescence, it does have some limitations. First, this study has used a broad measure to divide the participants into two groups. A positive result in CAPP indicates that a participant meets screening criteria for one or more of a range of possible disorders as clarified by DSM-IVText Revision. However, this broad grouping was employed because nonspecific emotional symptoms in adolescence have been shown to manifest as a range of disorders in early adulthood. ${ }^{2}$ Second, we have only investigated females given that depression is twice as prevalent in young women (aged 14-25 years) than men. ${ }^{32}$ This has likely reduced the generalisability of our findings, but importantly it has limited the potential confounding effect of gender and this is critical when first exploring new hypotheses. Third, we have considered a well-known triple-network model, but we acknowledge that there are other networks that may play an equally important role in emotion regulation and the essential development of emotional symptoms.

\section{Clinical implications}

The finding of a neural basis to the association between emotional symptoms and the reappraisal of negative emotions has the potential for profound clinical impact. Emotion dysregulation is an important transdiagnostic factor especially in adolescence that increases the risk for a wide range of psychopathology. ${ }^{33}$ Given that emotion regulation skills develop considerably throughout adolescence, and this is a period when regulatory neural circuitry is developing and taking shape. $^{34}$ This makes this phase of maturation an ideal period for testing and implementing psychological interventions targeting emotion regulation abilities. The identification of neural correlates is key to not only detecting changes predictive of psychopathology, but also for measuring improvement and quantifying the impact of treatment. However, before such exciting insights can help in curbing the development of full-blown emotional disorders in adulthood, it is necessary that these findings in adolescents are corroborated and mapped longitudinally over time.

\footnotetext{
Gin S. Malhi (D), Professor, Academic Department of Psychiatry, Northern Sydney Loca Health District: Advanced Research and Clinical High-Field Imaging, Sydney Medical School Northern, The University of Sydney; Discipline of Psychiatry, Sydney Medical school Northern, The University of Sydney; and CADE Clinic, Royal North Shore Hospital Northern Sydney Local Health District, Australia; Pritha Das, Senior Research Fellow, Academic Department of Psychiatry, Northern Sydney Local Health District; Advanced Research and Clinical High-Field Imaging, Sydney Medical School Northern, The University of Sydney; Discipline of Psychiatry, Sydney Medical School Northern, The University of Sydney; and CADE Clinic, Royal North Shore Hospital, Northern Sydney Local Health District, Australia; Tim Outhred, Postdoctoral Research Associate, Academic Department of Psychiatry, Northern Sydney Local Health District; Advanced Research and Clinical High-Field Imaging, Sydney Medical School Northern, The University of Sydney; Discipline of Psychiatry, Sydney Medical School Northern, The University of Sydney; and CADE Clinic, Royal North Shore Hospital, Northern Sydney LOcal Health District, Australia; Richard A. Bryant (O), Professor, School of PSych
}

New South Wales, Australia; Vince Calhoun, Professor, The Mind Research Network; and Department of Electrical and Computer Engineering, University of New Mexico, New Mexico, USA

Correspondence: Gin Malhi. Email: gin.malhi@sydney.edu.au

First received 28 May 2019, final revision 30 Oct 2019, accepted 31 Oct 2019

\section{Supplementary material}

Supplementary material is available online at https://doi.org/10.1192/bjp.2019.255.

\section{Acknowledgements}

This research is supported by an National Health and Medical Research Council programme grant (APP1073041) awarded to G.S.M., P.D. and R.A.B.

\section{Author contributions}

All authors contributed to the conception and design of the work, the interpretation of data, preparation of the manuscript and approval of the final work. In addition, P.D., T.O. and G.S.M. acquired neuroimaging and clinical data and P.D. conducted neuroimaging analysis. All authors contributed meaningfully to this research and remain accountable for all aspects of this publication.

\section{References}

1 Balazs J, Miklosi M, Kereszteny A, Hoven CW, Carli V, Wasserman C, et al. Adolescent subthreshold-depression and anxiety: psychopathology, func tional impairment and increased suicide risk. J Child Psychol Psychiatry 2013; 54(6): 670-7.

2 Shankman SA, Lewinsohn PM, Klein DN, Small JW, Seeley JR, Altman SE. Subthreshold conditions as precursors for full syndrome disorders: a 15-year longitudinal study of multiple diagnostic classes. J Child Psychol Psychiatry 2009; 50(12): 1485-94.

3 Fergusson DM, Horwood L, Ridder EM, Beautrais AL. Subthreshold depression in adolescence and mental health outcomes in adulthood. Arch Gen Psychiatry 2005; 62(1): 66-72.

4 Malhi GS, Das P, Outhred T, Bryant RA, Calhoun V. Resting-state neural network disturbances that underpin the emergence of emotional symptoms in adolescent girls: resting-state fMRI study. Br J Psychiatry 2019; 18: 1-7.

5 Cisler JM, Olatunji BO. Emotion regulation and anxiety disorders. Curr Psychiatry Rep 2012; 14(3): 182-7.

6 Gross JJ. Emotion regulation: affective, cognitive, and social consequences. Psychophysiology 2002; 39(3): 281-91.

7 Ochsner KN, Ray RD, Cooper JC, Robertson ER, Chopra S, Gabrieli JD, et al. For better or for worse: neural systems supporting the cognitive down- and upregulation of negative emotion. Neuroimage 2004; 23(2): 483-99.

8 Wang X, Feng Z, Zhou D, Lei X, Liao T, Zhang L, et al. Dissociable self effects for emotion regulation: a study of Chinese major depressive outpatients. BioMed Res Int 2014; 2014: 390865.

9 McLaughlin KA, Peverill M, Gold AL, Alves S, Sheridan MA. Child maltreatment and neural systems underlying emotion regulation. J Am Acad Child Adolesc Psychiatry 2015; 54(9): 753-62.

10 McRae K, Gross JJ, Weber J, Robertson ER, Sokol-Hessner P, Ray RD, et al. The development of emotion regulation: an fMRI study of cognitive reappraisal in children, adolescents and young adults. Soc Cogn Affect Neurosci 2012; 7(1): $11-22$. 
11 Miller AB, Prinstein MJ, Munier E, Machlin LS, Sheridan MA. Emotion reactivity and regulation in adolescent girls following an interpersonal rejection. J Cogn Neurosci 2019; 31(2): 249-61.

12 Stephanou K, Davey CG, Kerestes R, Whittle S, Pujol J, Yucel M, et al. Brain functional correlates of emotion regulation across adolescence and young adulthood. Hum Brain Mapp 2016; 37(1): 7-19.

13 Calhoun VD, Adalı T, Pekar JJ. A method for comparing group fMRI data using independent component analysis: application to visual, motor and visuomotor tasks. Magn Reson Imaging 2004; 22(9): 1181-91.

14 Calhoun VD, Kiehl KA, Pearlson GD. Modulation of temporally coherent brain networks estimated using ICA at rest and during cognitive tasks. Hum Brain Mapp 2008; 29(7): 828-38

15 Ambrosino S, Bos DJ, van Raalten TR, Kobussen NA, van Belle J, Oranje B, et al. Functional connectivity during cognitive control in children with autism spectrum disorder: an independent component analysis. J Neural Transm 2014; 121(9): 1145-55.

16 Menon V. Developmental pathways to functional brain networks: emerging principles. Trends Cogn Sci 2013; 17(12): 627-40.

17 Menon V. Large-scale brain networks and psychopathology: a unifying triple network model. Trends Cogn Sci 2011; 15(10): 483-506.

18 Manoliu A, Meng C, Brandl F, Doll A, Tahmasian M, Scherr M, et al. Insular dysfunction within the salience network is associated with severity of symptoms and aberrant inter-network connectivity in major depressive disorder. Front Hum Neurosci 2013; 7: 930.

19 Allen EA, ErhardtEB, Damaraju E, Gruner W, Segall JM, Silva RF, et al. A baseline for the multivariate comparison of resting-state networks. Front Syst NeurosCi 2011: 5: 2

20 Das P, Coulston CM, Bargh DM, Tanious M, Phan KL, Calhoun VD, et al. Neura antecedents of emotional disorders: a functional magnetic resonance imaging study of subsyndromal emotional symptoms in adolescent girls. Biol Psychiatry 2013; 74(4): 265-72.

21 Langsford S, Houghton S, Douglas G. The Child and Adolescent PsychProfile User Manual. Psychological and Educational Consultancy Services, 2004.

22 Kovacs M. The Children's Depression Inventory (CDI). Psychopharmacol Bull 1985; 21(4): 995-8.
23 Spielberger C. Manual for the State-Trait Anxiety Inventory. Consultant Psychologists Press, 1983

24 Gratz KL, Roemer L. Multidimensional assessment of emotion regulation and dysregulation: development, factor structure, and initial validation of the difficulties in emotion regulation scale. J Psychopathol Behav Assess 2004; 26(1): 41-54.

25 Lang PJ, Bradley MM, Cuthbert BN. International Affective Picture System (IAPS): Affective Ratings of Pictures and Instruction Manual. Technical Report A-8. University of Florida, Gainesville, FL, 2008.

26 Shirer WR, Ryali S, Rykhlevskaia E, Menon V, Greicius MD. Decoding subjectdriven cognitive states with whole-brain connectivity patterns. Cereb Cortex 2012; 22(1): 158-65.

27 Jafri MJ, Pearlson GD, Stevens M, Calhoun VD. A method for functional network connectivity among spatially independent resting-state components in schizophrenia. Neuroimage 2008; 39(4): 1666-81.

28 Falquez R, Couto B, Ibanez A, Freitag MT, Berger M, Arens EA, et al. Detaching from the negative by reappraisal: the role of right superior frontal gyrus (BA9/32). Front Behav Neurosci 2014; 8: 165

29 Balsters JH, Robertson IH, Calhoun VD. BOLD frequency power indexes working memory performance. Front Hum Neurosci 2013; 7: 207.

30 Baria AT, Baliki MN, Parrish T, Apkarian AV. Anatomical and functional assemblies of brain BOLD oscillations. J Neurosci 2011; 31(21): 7910-9.

31 Wang $X$, Zhou $X$, Dai $Q$, Ji $B$, Feng Z. The role of motivation in cognitive reappraisal for depressed patients. Front Hum Neurosci 2017; 11: 516

32 Albert PR. Why is depression more prevalent in women? J Psychiatry Neurosci 2015; 40(4): 219-21.

33 McLaughlin KA, Hatzenbuehler ML, Mennin DS, Nolen-Hoeksema S. Emotion dysregulation and adolescent psychopathology: a prospective study. Behav Res Therapy 2011; 49(9): 544-54.

34 Young KS, Sandman CF, Craske MG. Positive and negative emotion regulation in adolescence: links to anxiety and depression. Brain Sci 2019; 9(4): E76.

\section{poems by doctors}

\section{Postpartum Loneliness}

\section{Lois Holliday (iD}

Alone

Alone walking up the familiar route, next to you, each step as if never stepped before, the world around me getting smaller and falling into itself.

That nauseating feeling of unsettling newness, relentless. Unresolving. Unquenchable.

The watching from silent observers, judging, whispering. Always judging. Always there.

Crying and crying that incessant crying, unable to tell you when it has stopped or when he is calm

Unable to pacify

That incessant crying.

Getting up, checking, rechecking

What's that noise? Checking.

Unable to move, unable to stop, restless motionless silence and that continual crying.

Unable to tell you or explain, unable to know.

Can no one tell that I am not okay?

The crippling terror of the judgement, the fear, the guilt.

Voiceless screaming, actionless begging, whilst smiling, carrying on and pouring out tea

Us both crying

Alone. 